\title{
Fatigue Responses in Various Muscle Groups in Well-Trained Competitive Male Players after a Simulated Soccer Game
}

\author{
by \\ Dan Fransson ${ }^{1}$, Jeppe Foged Vigh-Larsen ${ }^{2}$, Ioannis G. Fatouros ${ }^{3}$, Peter Krustrup ${ }^{4,5}$, \\ Magni Mohr ${ }^{1,4,6}$
}

We examined the degree of post-game fatigue and the recovery pattern in various leg and upper-body muscle groups after a simulated soccer game. Well-trained competitive male soccer players $(n=12)$ participated in the study. The players completed the Copenhagen Soccer Test, a $2 \times 45$ min simulated soccer protocol, following baseline measures of maximal voluntary contractions of multiple muscle groups and systemic markers of muscle damage and inflammation at 0, 24 and $48 \mathrm{~h}$ into recovery. All muscle groups had a strength decrement $(p \leq 0.05)$ at $0 \mathrm{~h}$ post-match with knee flexors $(14 \pm 3 \%)$ and hip abductors $(6 \pm 1 \%)$ demonstrating the largest and smallest impairment. However, $24 \mathrm{~h}$ into recovery all individual muscles had recovered. When pooled in specific muscle groups, the trunk muscles and knee joint muscles presented the largest decline $0 \mathrm{~h}$ post-match, $11 \pm 2 \%$ for both, with the performance decrement still persistent $(4 \pm 1 \%, p \leq 0.05)$ for trunk muscles $24 \mathrm{~h}$ into recovery. Large inter-player variations were observed in gameinduced fatigue and recovery patterns in the various muscle groups. Markers of muscle damage and inflammation peaked $0 \mathrm{~h}$ post-match (myoglobin) and $24 \mathrm{~h}$ into recovery (creatine kinase), respectively, but thereafter returned to baseline. Intermittent test performance correlated with creatine kinase activity $24 \mathrm{~h}$ after the Copenhagen Soccer Test ( $r$ $=-0.70 ; p=0.02)$. In conclusion, post-game fatigue is evident in multiple muscle groups with knee flexors showing the greatest performance decrement. Fatigue and recovery patterns vary markedly between muscle groups and players, yet trunk muscles display the slowest recovery.

Key words: muscle fatigue, recovery, muscle damage, inflammation, soccer.

\section{Introduction}

A soccer game is characterized by an activity pattern with frequent changes, prolonged intermittent exercise conjoined with short highintensity running bouts and explosive actions such as accelerations, decelerations, change of direction, jumps, and tackles demanding a high rate of force development (Varley and Aughey, 2012). During a soccer game, the energy demands are high, and both aerobic and anaerobic energy sources are greatly utilized (Krustrup et al., 2006b), which is likely to affect exercise performance.

A major research topic during the last two decades has been game-induced fatigue in soccer. Match analysis studies have demonstrated performance decrements during and towards the

\footnotetext{
1 - Center for Health and Performance, Department of Food and Nutrition, and Sport Science, University of Gothenburg,

Gothenburg, Sweden.

2- Section for Sport Science, Department of Public Health, Aarhus University, Aarhus, Denmark.

3 - School of Physical Education and Sport Science, University of Thessaly, Karies, Trikala, Greece.

4- Department of Sports Science and Clinical Biomechanics, SDU Sport and Health Sciences Cluster (SHSC), Faculty of Health Sciences, University of Southern Denmark, Odense, Denmark.

5 - Sport and Health Sciences, College of Life and Environmental Sciences, St. Luke's Campus, University of Exeter, Exeter, United Kingdom.

6. Centre of Health Science, Faculty of Health Sciences, University of the Faroe Islands, Tórshavn, Faroe Islands.
} 
end of competitive games (Fransson et al., 2017; Mohr et al., 2003) and several studies have shown impairment of whole-body exercise performance, such as jumping, sprinting and high intensity running, after a game (Bangsbo et al., 2007). In support of this, studies that have applied postgame strength testing of single muscle groups, such as the quadriceps, report game-induced impairment in muscle function (Krustrup et al., 2011). Moreover, physiological measurements obtained after a game demonstrate a marked decrease in muscle glycogen pools located in subcellular compartments, attenuated $\mathrm{Ca} 2+$ (Krustrup et al., 2011) and a rise in systemic markers of muscle damage, inflammation, and oxidative stress (Mohr et al., 2016), all of which have been linked to muscle fatigue (Bangsbo et al., 2007; Magalhaes et al., 2010; Mohr et al., 2003). Thus, there is solid evidence in the literature that soccer players experience fatigue, muscle damage, and inflammation after a soccer game, and that performance impairment appears to have a slow recovery rate (Andersson et al., 2008; Ispirlidis et al., 2008). However, since the majority of studies investigating muscle function after a soccer game have examined the quadriceps muscle, it is currently unknown whether different muscle groups of importance for soccer performance display different fatigue and recovery patterns after a game. In a few studies, the game-induced effect has been assessed in both knee flexors and extensors, and it appears that these muscles are affected differently by team sports (soccer, basketball, handball) incorporating sprinting, cutting, and jumping actions (Chatzinikolaou et al., 2010, 2014; Draganidis et al., 2015; Mohr et al., 2016). Since the highly intermittent movement pattern during a soccer game is likely to involve all muscle groups in the lower limbs as well as core muscles, it is important to understand the effect of a soccer game on different muscle groups during post-game recovery.

The fatigue response in a given muscle group during team sports such as soccer is likely to be affected by a player's individual movement style during a game, training status, muscle morphology, motor skills, and the degree of balance in strength between agonist, antagonist, and synergist muscles. It is well known that there are major inter-player variations in high-intensity activity during a game (Fransson et al., 2017), as well in physical performance (Krustrup et al., 2006a). Thus, it is likely that players may display a different fatigue and recovery response in different muscle groups after a game.

The work rate in a soccer game is influenced by numerous factors, such as technical quality, tactical role, opponent, playing style, etc., which cause large inter-game variability (Gregson et al., 2010). Thus, examination of physical phenomena may be affected by other than physiological factors. The Copenhagen Soccer Test (CST) was therefore developed as a standardized exercise protocol simulating the physical and physiological load of a soccer game (Bendiksen et al., 2012). In the study by Bendiksen and colleagues (2012), the CST was shown to produce a similar external and internal load as a competitive game and may therefore be a highly suitable standardized model for investigating post-game fatigue and a recovery pattern in soccer.

Thus, the aim of the present study was to examine the degree of fatigue and the recovery pattern in different muscle groups in competitive players after a simulated soccer game. Welltrained male players completed the CST, and performance was measured in various muscle groups after the game and during the first two days of recovery. We hypothesized that: 1) a muscle-group-specific decline in performance would occur after the CST; 2) particular muscle groups would recover differently during the first few days after a game; and that 3 ) muscle-specific fatigue and recovery patterns are highly individual.

\section{Methods}

To investigate the recovery response of multiple muscle groups after a standardized workload resembling a competitive soccer match, well-trained players performed a simulated soccer protocol, the CST, following baseline measurements of muscle function and blood markers of muscle damage and inflammation. Subsequently, baseline measures were repeated at 0,24 and $48 \mathrm{~h}$ to evaluate the degree of postmatch fatigue and the recovery pattern of individual muscle groups. To reduce variability, the CST was completed twice for each player following $72 \mathrm{~h}$ of recovery, when full recovery had been achieved. Prior to completion of the 
CST, additional fitness tests were conducted to assess the physical capacity of the players and enable correlations between the fitness level and post-match fatigue response.

\section{Participants}

Competitive male soccer players $(n=12)$ from the Swedish second and third divisions (age: $23 \pm 1$ yrs; body height: $180 \pm 2 \mathrm{~cm}$; body mass: 75 $\pm 2 \mathrm{~kg} ; \mathrm{VO}_{2 \max }: 61 \pm 1 \mathrm{mlO} \cdot \mathrm{min}^{-1} \cdot \mathrm{kg}^{-1}$; Yo-Yo intermittent recovery level 2 test (Yo-Yo IR2) performance: $927 \pm 40 \mathrm{~m}$ ) participated in the study. All participants volunteered and consented to take part in the study. The inclusion criteria were Yo-Yo IR2 performance of minimum $800 \mathrm{~m}$ (Krustrup et al., 2006a) and free of injury for at least 6 weeks prior to testing. The players had a weekly training schedule of 6-7 sessions. The study was approved by the local ethics committee and conducted in accordance with the Declaration of Helsinki (1964). After being informed verbally and in writing of the experimental procedures and associated risks, all participants gave their written consent to participate in the study.

\section{Measures}

A within-subject design was used to longitudinally compare the fatigue responses and recovery kinetics in different muscle groups during and after a simulated soccer match. Thus, two simulated soccer matches separated by $72 \mathrm{~h}$ of recovery were completed using the CST (Bendiksen et al., 2012). In the week preceding the match simulations, players carried out $\mathrm{VO}_{2 \max }$ and Yo-Yo IR2 testing on separate days. The participants were instructed to refrain from strenuous exercise and intake of alcohol for $48 \mathrm{~h}$, and of caffeine and tobacco for $12 \mathrm{~h}$, prior to testing and before and during the week of the match simulations. Likewise, they were instructed to follow and observe their usual diet before all testing sessions and during the study in order to optimize standardized conditions. The players did not train on recovery days to avoid the impact of additional loading during the initial $48 \mathrm{~h}$ of recovery. The CST was preceded and succeeded by blood sampling and maximal voluntary contraction (MVC) measurements for multiple muscle groups (knee flexors, knee extensors, ankle extensors, hip adductors, hip abductors, lumbar/thoracic flexors, lumbar/thoracic extensors, and lumbar/thoracic rotators) at baseline and 0,24 and $48 \mathrm{~h}$ into the recovery phase. This enabled to differentiate fatigue and recovery between various muscle groups. To familiarize the subjects with the measurements and determine the individual configurations of the equipment, the subjects completed one preexperimental session in the week preceding the study. The CST was conducted between 9:30 am and 2:30 pm, and the time of day was kept constant for each subject. The players were familiarized with the CST prior to the study. Two CST trials were completed for each player in order to reduce variability, and mean values from both testing trials were used as the test result. On test days, the first subject reported to the laboratory at 7:30 am and had a baseline blood sample collected before conducting the baseline MVC tests for all the muscle groups investigated. Subsequently, the CST was carried out as previously described by Bendiksen et al. (2012). The test consists of $2 \times 45 \mathrm{~min}$ of soccer-specific movements separated by a 15-min break, reflecting the activities of a real competitive match. After the simulated match, another blood sample was drawn and another MVC testing session completed in order to analyze blood markers of muscle damage and inflammation and to evaluate the strength loss of the muscles. Throughout the CST, the heart rate was measured continuously and the activity pattern determined by the GPS, as described by Randers et al. (2010). Furthermore, capillary blood samples were collected during the warm-up and at 15-min intervals throughout the match and analyzed for levels of lactate. This was done at the end of each 5 -min period during a 15-30-s scheduled rest period before continuing the next period. In addition, a 20-m shuttle sprint test was carried out every 5 min during the test.

\section{Design and Procedures}

The CST simulated soccer match used in the study is a field test mimicking the activities of a real competitive elite soccer match based on the activity patterns observed in the study by Mohr et al. (2003). The mean duration and mean distances covered in each different movement category as well as the time in possession of the ball and the number of jumps, shots, and passes were all incorporated in the test (Bendiksen et al., 2012). The physiological response to the CST is reproducible and comparable to that of a highlevel competitive soccer match. Thus, the CST 
includes various actions, including running at different speeds and in various directions (e.g. backwards, sideways), sprinting, jumping, changing direction, and ball actions such as dribbling, shooting, and passing. The test is divided into 18 periods of approximately $5 \mathrm{~min}$ each varying in intensity between low (L), medium (M), and high $(\mathrm{H})$. As such, each half consists of 95 -min periods in the sequence L-MH-L-M-M-L-M-H, the 5-min bouts representing $152,171,69,41,55,40,30$, and $23 \mathrm{~m}$ of walking $(\sim 6$ $\mathrm{km} / \mathrm{h})$, jogging $(\sim 8 \mathrm{~km} / \mathrm{h})$, low-speed running $(\sim 12$ $\mathrm{km} / \mathrm{h})$, moderate-speed running $(\sim 15 \mathrm{~km} / \mathrm{h})$, highspeed running $(18 \mathrm{~km} / \mathrm{h})$, sprinting $(>25 \mathrm{~km} / \mathrm{h})$, backwards running $(\sim 10 \mathrm{~km}), \quad$ and backwards/sideways running $(\sim 8 \mathrm{~km} / \mathrm{h})$, respectively. In the medium- and high-intensity periods, additional 20 and $60 \mathrm{~m}$ of moderatespeed running and 22 and $65 \mathrm{~m}$ of high-speed running are added, respectively. Technical tests and a shuttle sprint test are incorporated in the CST. During each 5-min period, the subjects have $10 \mathrm{~s}$ to take a free-kick aiming at the goal from the edge of the penalty area, achieving points for accuracy. Likewise, a passing test is performed, followed by a $2 \times 20-\mathrm{m}$ shuttle sprint test in each 5 -min bout. However, as the data from the shooting and passing tests were not included in the analysis of this study, these tests are not described in detail. Subjects had individual water bottles prepared and were allowed to drink 1 liter of water during the test. Experienced test personnel supervised the subjects constantly during the test and speeds were controlled by sound emission. Prior to each CST protocol, a 15min warm-up was conducted, as previously described (Bendiksen et al., 2012). Familiarization preceded experimental testing.

A repeated sprinting test (RSA) was conducted after the warm-up consisting of five $2 x$ $20-\mathrm{m}$ shuttle sprints with $30 \mathrm{~s}$ of rest in between. The sprints were initiated from a standing position $50 \mathrm{~cm}$ behind the first timing gate, with the players sprinting $20 \mathrm{~m}$ in a straight line before turning at a marked line and sprinting all the way back through the timing gates. Furthermore, at the end of each 5-min period during the CST a single $2 \times 20-\mathrm{m}$ shuttle sprint was performed in the same manner. Players were instructed to sprint at maximal speed and verbally encouraged during each effort. Sprint times were recorded using Muscle Lab V8 (Bosco System, Rome, Italy) photocells with precision of $0.001 \mathrm{~s}$.

Blood samples were taken from an antecubital vein in the right arm using flexible Venflon cannulas at baseline, immediately after the match, and every $24 \mathrm{~h}$ during the recovery with subjects seated. Venous blood was drawn in vacutainer EDTA tubes and serum separation tubes. EDTA tubes were centrifuged immediately at $4^{\circ} \mathrm{C}$ and plasma stored at $-80^{\circ} \mathrm{C}$ until analysis. Blood in serum separation tubes was allowed to coagulate at room temperature, centrifuged and serum stored at $-80^{\circ} \mathrm{C}$ until analysis. Blood samples taken during the CST were collected from a fingertip to measure capillary blood lactate and analyzed using a Biosen analyzer (Biosen C-line, EKF-diagnostic GmbH, Magdeburg, Germany). These were collected at rest, after the warm-up, before the first half (after the RSA), after 15, 30, and $45 \mathrm{~min}$ of the first half, before the second half, and after 15, 30, and $45 \mathrm{~min}$ of the second half.

The HR was recorded at 5-s intervals during the CST using Polar HR monitors with a chest strap. Activity patterns were recorded by the GPS using Catapult Sport OPTIMEYE S5 with a sampling rate of $15 \mathrm{~Hz}$ (Randers et al., 2010). Both types of data were stored on a computer for later analysis using Catapult sprint software 5.1.7 for team sports.

Maximal voluntary isometric contraction (MVC) was determined for all examined muscle groups. The David system F300 (David, Outokumpu, Finland) was used for all muscle groups except the ankle extensors, which were tested using Isomed 2000 (D\&R Ferstl, Hernau, Germany). Each participant visited the laboratory a week prior to the study to undergo a familiarization session and have their individual configurations determined, such as foot- and backrest settings. The warm-up procedure during MVC measurements before the simulated matches and during recovery days consisted of $10 \mathrm{~min}$ of jogging at $10 \mathrm{~km} / \mathrm{h}$ and four repetitions of lumbar/thoracic rotations on each side with $30 \mathrm{~kg}$ of resistance. After the CST, no further warm-up was performed, as the subjects immediately walked back to the laboratory $(\sim 400 \mathrm{~m})$ for MVC testing. Tests were performed for both the dominant and non-dominant leg in randomized order, and the order of muscle groups tested was also randomized and kept constant for each 
subject during the tests. The subjects performed three maximal trials (five for hip abductors and adductors due to an activation effect predominantly for these movements), eliciting force for 3-5 s with $45 \mathrm{~s}$ of rest between trials. For all measurements, subjects were instructed not to use their hands for extra support and to put on a waist strap. Experienced test personnel supervised the participants and supported them with verbal encouragement. No visual feedback was provided during the tests and the highest peak torque was used for further analysis.

\section{Statistical Analysis}

Data are presented as mean \pm SEM. A Shapiro-Wilk test was used to test data normality. The mean MVC value for trials 1 and 2 for each player was analyzed using a one-way ANOVA for repeated-measures analysis of variance. Sprint times throughout each simulated game, lactate values, and blood samples in the recovery phase were also averaged between trial 1 and 2 and likewise analyzed using a one-way ANOVA for repeated-measures analysis of variance. When a significant interaction was found, multiple comparison procedures were applied using a Tukey post hoc test to identify the points of difference. Significance was accepted at $p \leq 0.05$. Correlations were calculated between fitness, blood, and GPS data, and the percentage decrement of MVC $0 \mathrm{~h}$ post-match using a Pearson Product Moment Correlation. SigmaStat was used for all analyses (SigmaStat for Windows version 11.0).

\section{Results}

\section{Muscle-specific performance}

All investigated muscle groups had a significant decline in MVC torque immediately after $(0 \mathrm{~h}$ post $)$ the simulated soccer match $(p \leq$ $0.05)$ (Figure 1A). The largest percentage decline, $14 \pm 3 \%$, was observed for knee flexors (198 \pm 7 vs. $171 \pm 12 \mathrm{Nm}$ ), followed by $12 \pm 1 \%$ for lumbar/thoracic extensors (353 \pm 15 vs. $308 \pm 12$ $\mathrm{Nm}), 10 \pm 2 \%$ for lumbar/thoracic flexors (212 \pm 8 vs. $191 \pm 10 \mathrm{Nm}), 9 \pm 4 \%$ for lumbar/thoracic rotators (203 \pm 6 vs. $184 \pm 9 \mathrm{Nm}$ ), $9 \pm 2 \%$ for hip adductors ( $437 \pm 38$ vs. $405 \pm 41 \mathrm{Nm}$ ), $9 \pm 2 \%$ for ankle extensors (191 \pm 10 vs. $175 \pm 10 \mathrm{Nm}$ ), $8 \pm 2 \%$ for knee extensors $(240 \pm 14$ vs. $219 \pm 14 \mathrm{Nm})$, and $6 \pm 1 \%$ for hip abductors (315 \pm 14 vs. $296 \pm 14$ $\mathrm{Nm})$. However, $24 \mathrm{~h}$ into recovery there were already no differences in MVC in the individual muscles compared to baseline values. The different players displayed large individual differences in fatigue and recovery responses in the various muscle groups (Figure 1C).

When pooled in specific categories of muscles, all muscle groups showed a significant $(p$ $\leq 0.05$ ) strength loss $0 \mathrm{~h}$ post CST, with the largest decrease for knee joint muscles (combined knee flexors and extensors) and trunk muscles (combined lumbar/thoracic extensors, rotators, and flexors), both $11 \pm 2 \%$ (376 \pm 24 vs. $349 \pm 26$ and $258 \pm 8$ vs. $229 \pm 7 \mathrm{Nm}$ ), and the smallest decrease for hip joint muscles (hip abductors and adductors), $8 \pm 1 \%$ ( $p \leq 0.05$; Figures $1 \mathrm{~B}$ and 1D). Additionally, a decline in strength remained $24 \mathrm{~h}$ post match for the trunk muscles, $4 \pm 1 \%$ (258 \pm 8 vs. $248 \pm 9 \mathrm{Nm}, p \leq 0.05)$, with all other muscle groups recovered.

Table 1

Match activities during the Copenhagen Soccer Test (CST)

\begin{tabular}{ll}
\hline Activity variables & Mean \pm SEM \\
\hline Total distance covered $(\mathrm{m})$ & $11283 \pm 121$ \\
Distance $(\mathrm{m})$ in speed zone $0-11 \mathrm{~km} / \mathrm{h}$ & $7655 \pm 70$ \\
Distance $(\mathrm{m})$ in speed zone $11-14 \mathrm{~km} / \mathrm{h}$ & $834 \pm 78$ \\
Distance $(\mathrm{m})$ in speed zone $14-17 \mathrm{~km} / \mathrm{h}$ & $745 \pm 56$ \\
Distance $(\mathrm{m})$ in speed zone $17-21 \mathrm{~km} / \mathrm{h}$ & $1457 \pm 51$ \\
Distance $(\mathrm{m})$ in speed zone $21-24 \mathrm{~km} / \mathrm{h}$ & $531 \pm 66$ \\
Distance $(\mathrm{m})$ in speed zone $24-40 \mathrm{~km} / \mathrm{h}$ & $62 \pm 23$ \\
Number of accelerations above $2 \mathrm{~m} / \mathrm{s}^{2}$ & $49 \pm 14$ \\
Number of decelerations above $2 \mathrm{~m} / \mathrm{s}^{2}$ & $38 \pm 11$ \\
\hline
\end{tabular}



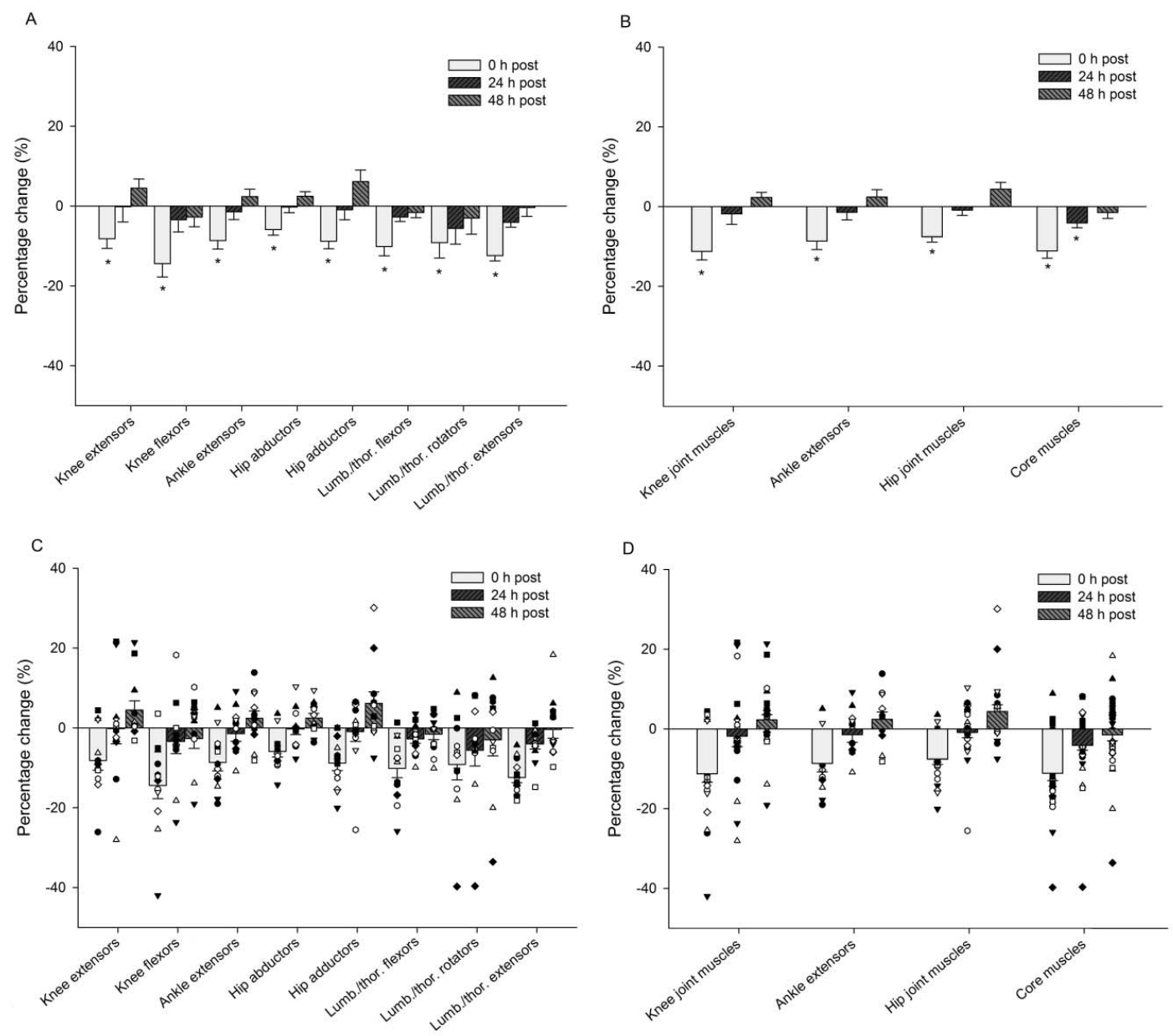

Figure 1.

Post-game MVC results in individual muscle groups (A), pooled in specific muscle categories

(B). Data is presented as mean values and standard deviation.

$C$ shows individual results in individual muscle groups and

$D$ individual results in pooled specific muscle categories.

* Significantly different from baseline at $p \leq 0.05$. 

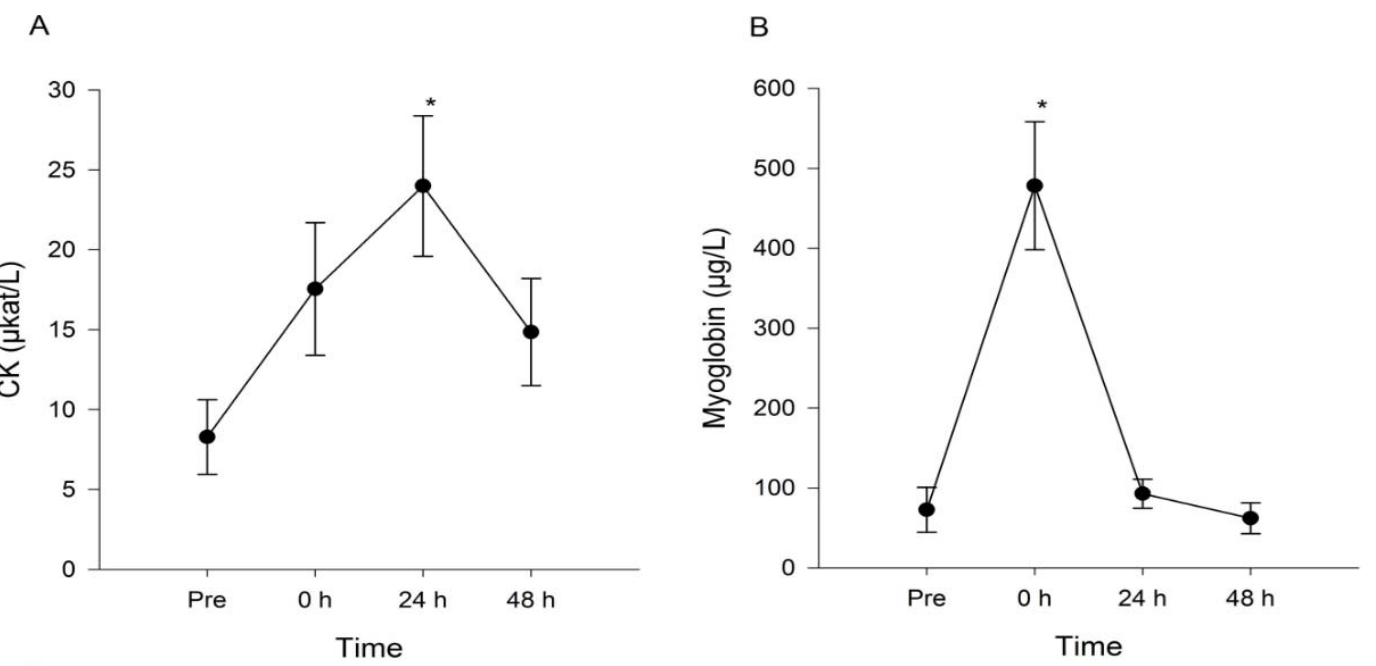

C

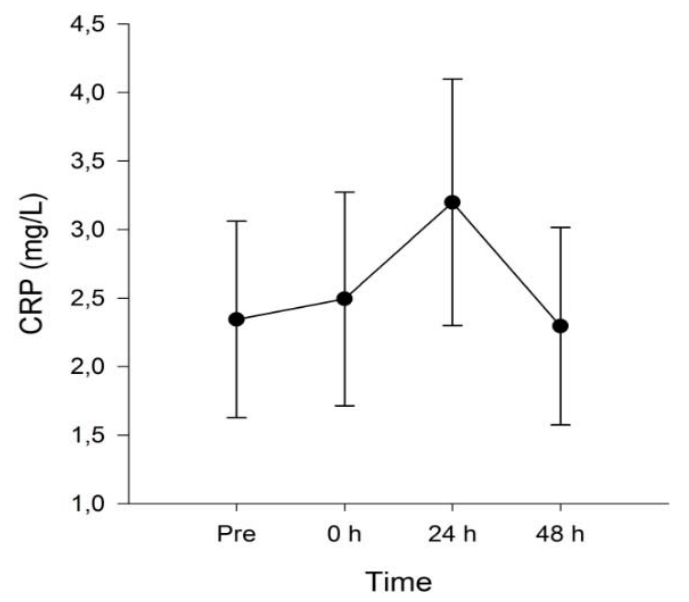

Figure 2

Muscle damage markers (CK) (A), myoglobin (B) and inflammatory response $(C R P ; C)$ following the Copenhagen Soccer Test (CST).

*Significantly different from baseline at $p \leq 0.05$. 

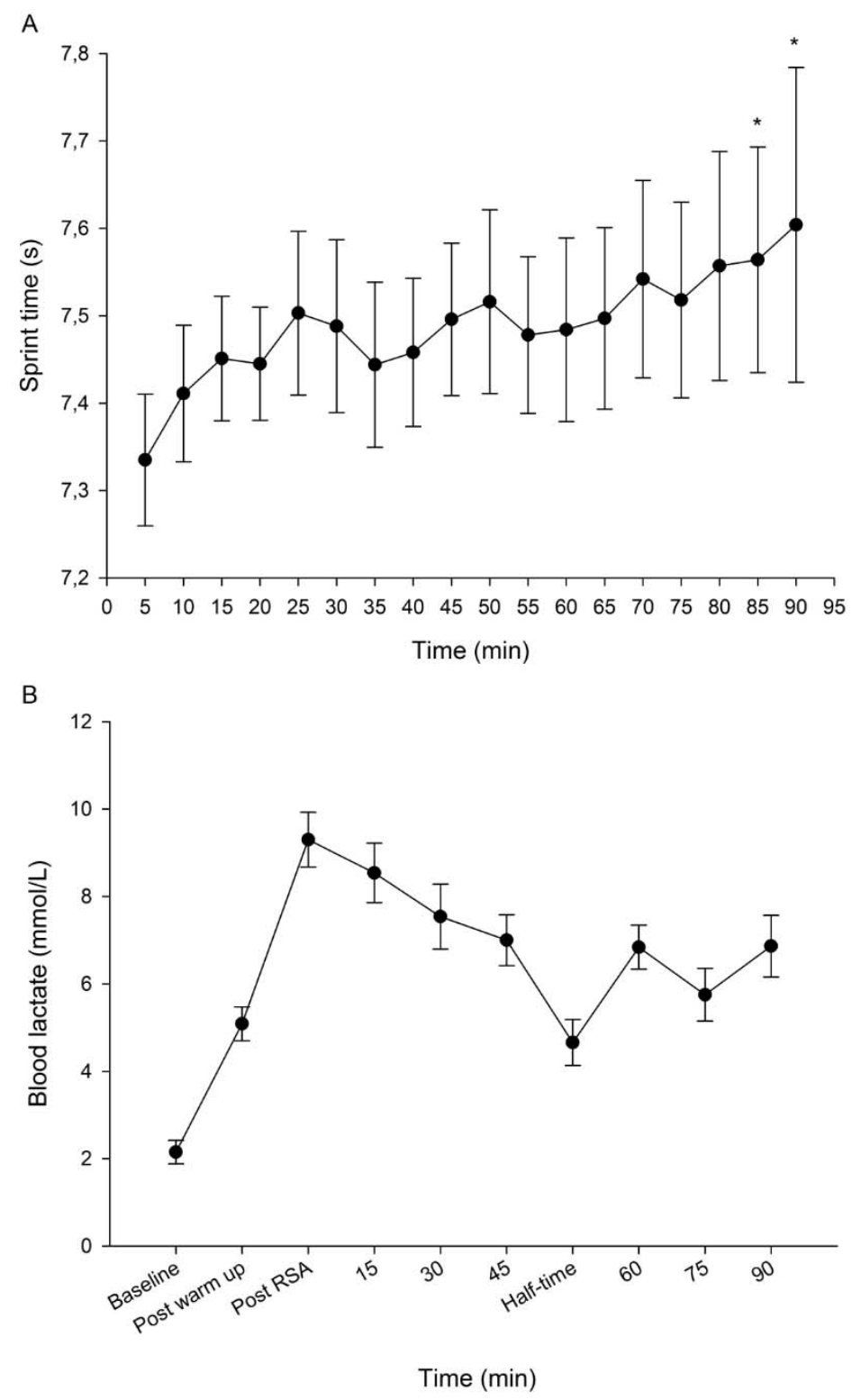

Figure 3

$2 \times 20 \mathrm{~m}$ shuttle sprint performance (A) and capillary blood lactate

(B) during the Copenhagen Soccer Test (CST).

*Significantly different from baseline at $p \leq 0.05$.

Markers of muscle damage and inflammation

Plasma CK activity increased threefold 24

$\mathrm{h}$ after the match $(p \leq 0.05)$ and returned to baseline after $48 \mathrm{~h}$ of recovery (Figure 2A). Myoglobin concentration increased $(p \leq 0.05)$ sevenfold immediately after the match $(0 \mathrm{~h}$ post $)$ 
but returned to baseline $24 \mathrm{~h}$ into recovery (Figure 2B). No significant changes were observed for concentrations of CRP, which remained stable at all time points throughout recovery (Figure 2C).

\section{Physical and physiological response to the CST}

Table 1 presents the GPS-measured activity profile during the CST. The activities resulted in a mean heart rate of $82 \pm 1 \% \mathrm{HR}_{\max }$, with peak values of $97 \pm 1 \% \mathrm{HR}_{\max } \mathrm{CV}$ values for total distance and distance in speed zones 0-11, 11-14, 14-17, 17-21, 21-24, and $>24 \mathrm{~km} \cdot \mathrm{h}^{-1}$ for the first and second CST completed by each subject were $0.7,1.1,11.5,16.2,7.1,22.4$, and $66.0 \%$, respectively. Sprint time during the first $2 \times 20-\mathrm{m}$ sprint was $7.335 \pm 0.075 \mathrm{~s}$, which was significantly $(p \leq 0.05)$ faster than the sprint time during the final sprint $90 \mathrm{~min}$ into the CST $(7.604 \pm 0.181 \mathrm{~s}$; Figure 3A). Likewise, there was a tendency ( $p=$ $0.08)$ toward a significant difference between the first and 17th sprints $(7.335 \pm 0.075 \mathrm{~s}$ vs. $7.564 \pm$ $0.129 \mathrm{~s})$ after $85 \mathrm{~min}$. Blood lactate was $2.1 \pm 0.3$ $\mathrm{mmol} / \mathrm{L}$ at baseline and higher at all time points $(p$ $\leq 0.05)$, reaching $8.5 \pm 0.7 \mathrm{mmol} / \mathrm{L} 15 \mathrm{~min}$ into the CST, but lowering to $6.9 \pm 0.7 \mathrm{mmol} / \mathrm{L}$ after $90 \mathrm{~min}$ $(p \leq 0.05$; Figure 3B). Overall, the blood lactate levels were higher in the first than in the second half $(7.7 \pm 0.6$ vs. $6.3 \pm 0.6 \mathrm{mmol} / \mathrm{L} ; p \leq 0.05)$.

\section{Correlations}

There was a positive relationship between YoYo IR2 performance and the CK activity change score $24 \mathrm{~h}$ after the CST $(\mathrm{r}=-0.70 ; p=0.02)$. In contrast, no significant correlations were observed between Yo-Yo IR2 performance and the decrease in MVC in any muscles after the test. There was a strong tendency for an inverse correlation between the percentage drop in sprint time during the final sprint and the percentage drop in MVC in the hamstrings muscles $(\mathrm{r}=-0.63 ; p=$ 0.052). Additionally, the drops in MVC in the hamstrings ( $\mathrm{r}=0.63 ; p=0.03)$ and adductor muscles $(\mathrm{r}=0.62 ; p=0.03)$ were correlated with body mass of the players.

\section{Discussion}

The present study is the first to examine differences in fatigue response between multiple muscle groups and recovery kinetics after a simulated soccer match. The principal findings were that: i) fatigue occurred in multiple muscle groups after a simulated soccer game, with knee joint and trunk muscles experiencing the largest decline; ii) large individual responses were observed between players in fatigue and recovery kinetics; and iii) physical capacity correlated to markers of muscle damage $24 \mathrm{~h}$ after the game.

Our findings showed that all the examined muscle groups displayed a decline in MVC torque immediately after the CST. Thus, the type of post-game fatigue previously demonstrated in match analysis studies (Fransson et al., 2017; Mohr et al., 2003) as well as in studies applying whole-body post-game testing protocols (Krustrup et al., 2006b; Mohr et al., 2016) appears to be induced by fatigue in several muscle groups. The finding of game-induced decrement in muscle strength is in accordance with numerous studies primarily investigating the knee extensors and flexors after soccer games or simulated models (Delextrat et al., 2013; Draganidis et al., 2015; Krustrup et al., 2011). These studies likewise demonstrate a reduction in MVC performance of $9-15 \%$ immediately after a game, which is comparable to the 14 and $8 \%$ decrement in knee flexors and extensors in the present study. In addition, Nybo et al. (2013) and Girard et al. (2015) measured MVC in the ankle extensors, observing no decrease immediately post-match (non-significant $5 \%$ decreases) or during the recovery phase, contrasting with our finding of a $9 \%$ decrease.

In our study, knee flexors displayed the largest game-induced decline $(14 \%)$ in muscle strength, followed by several of the trunk muscles (9-12\%), while the post-game impairment of knee flexor strength was $8 \%$. However, when pooled in specific categories of muscles, all muscle groups displayed a fatigue response, with the largest decline for knee joint muscles (combined knee flexors and extensors) and trunk muscles (combined lumbar/thoracic extensors, rotators and flexors), both $11 \%$, and the smallest decrease for hip joint muscles (hip abductors and adductors), $8 \%$. Similar results for knee flexors are reported in studies using both simulated soccer play and actual games (Delextrat et al., 2013; Draganidis et al., 2015). Specifically, soccer play seems to leave the concentric strength of knee flexors unaffected (Delextrat et al., 2013) and reduce the eccentric strength of this muscle group (Draganidis et al., 2015). The hamstrings contract eccentrically in cutting maneuvers, kicking, tackling, sprinting, etc., to counteract anterior 
shear forces generated by knee extensors, decelerate lower-limb motion before foot contact, and protect the knees from hyperextension (Hawkins et al., 2001). These results suggest that fatigue may compromise the capability of knee flexors to stabilize the knee during the first $24 \mathrm{~h}$ of game recovery, thereby placing more stress on the ACL. Additionally, a significant decline in strength was still observed $24 \mathrm{~h}$ post-match in the trunk muscles, $4 \%$, with all other muscle groups recovered. This is not surprising, since the muscles of the trunk serve as a stable base not only for limb movement but also for force transfer. In soccer, most on-pitch activities include intense running and rotational movements. Highintensity running, collisions, and cutting maneuvers incorporate continuous hip flexion and extension motions that exert destabilizing torques on the body core, and the trunk muscles need to contract to form a stable base for the moving limbs (Behm et al., 2009). The trunk muscles contract together to resist rotational forces and provide stability for spinal stabilization (Shinkle et al., 2012).

The players displayed large individual differences in fatigue and recovery response in the various muscle groups. For example, one player had a very large strength loss of $41 \%$ for the knee flexors immediately post-match, the strength remaining $21 \%$ below baseline $48 \mathrm{~h}$ into recovery, by contrast with another player who displayed no decrease in strength at any time points for this particular muscle group (Figure 3C). For the hip adductors, one player had a strength loss immediately after the match of $20 \%$, the strength remaining $7 \%$ from pre-game values $48 \mathrm{~h}$ into recovery, with other players showing only minor changes. This pattern of individual variation was similar for all the investigated muscles. Thus, despite no performance deficits being visible overall, some individuals could have a prolonged recovery of performance, with some specific muscle groups possibly being more vulnerable than others. As such, this could provide valuable information in a team setting, where coaches should try to identify individual differences and implement training and rotation strategies based on these observations. However, the evaluation of individual differences has to be carried out cautiously, as random variability, error of measurements, etc., could influence these results.
Several data points should therefore be collected for the same individual before drawing specific conclusions. Thus, future research should focus on gaining more knowledge on individual differences in recovery in high-level soccer players.

In general, the literature contains conflicting results regarding the time needed to allow recovery of muscle strength after soccer matches. Recovery duration ranges from $24 \mathrm{~h}$ in the observations by Silva et al. (2013) to $48 \mathrm{~h}$ as described by Rampinini et al. (2011) and Krustrup et al. (2011), and even longer, $72 \mathrm{~h}$, in other studies (Ascensão et al., 2008; Draganidis et al., 2015; Nedelec et al., 2014). The results of the present study are in line with those obtained by Silva et al. (2013), with the overall pattern of recovery showing no significant declines just $24 \mathrm{~h}$ post-match simulation. The discrepancy in the literature could be related to the presence or absence of daily soccer practice during the recovery period. For example, players trained every day during the post-match period in the studies by Nedelec et al. (2014) and Draganidis et al. (2015), possibly contributing to the prolonged decrease in strength observed in these studies. Accordingly, Draganidis et al. (2015) found a reduction in MVC after the most intense weekly training session for a control group only participating in training. High-level players are likely to recover faster than lower-level players due to their higher training status. In the studies by Ascensão et al. (2008) and Magalhaes et al. (2010), players were instructed to refrain from exhaustive exercise during the recovery period, but these were lower-level players, which may partly explain the prolonged recovery observed for these players. The type of a match, e.g. friendly or competitive, may influence the demands on the players. In the present study, we used a simulated soccer model (CST), which might have affected the load on the players and potentially caused a less pronounced strength loss following the match simulations. However, the CST had previously been validated by Bendiksen et al. (2012) showing a physiological response analogous to that of a competitive game for the same players. Moreover, the CST produced a similar elevation of the HR (86\% HRmax) and blood lactate concentration $(\sim 7 \mathrm{mM}$, with the average level higher in the first than in the second 
half), suggesting that this simulated soccer play produced similar physiological and metabolic responses to those usually observed in actual competitive soccer play (Fatouros et al., 2010; Ispirlidis et al., 2008; Mohr et al., 2016) and was able to elicit a strain in players usually seen in actual match play (Krustrup et al., 2006a; Mohr et al., 2016).

The decline in muscle strength and the rise in CK activity suggest that the CST induced muscle damage of a relatively short timeframe (24 h). The magnitude of the increase in CK activity was comparable to that usually seen in response to competitive soccer games (Mohr et al., 2016; Silva et al., 2013). The elevation in CK activity in the circulation is related to its augmented efflux from muscle, due to the increased permeability of muscle-fiber membrane, and/or from intramuscular vasculature (Fatouros and Jamurtas, 2016). The faster recovery seen following the CST as compared to competitive games may be related to greater distances covered by high-intensity running, sprinting, and accelerating/decelerating. In addition, the lack of physical contact between players in a simulated model may also have played a role. Draganidis et al. (2015) reported that muscle damage responses in soccer were significantly related to on-pitch activity during the game and, specifically, to sprinting and accelerations/decelerations. The CST also induced a short-lived inflammatory response, as evidenced by the CRP values (exceeded $3 \mathrm{mg} / \mathrm{L}$ ), a response also seen after actual match play (Ispirlidis et al., 2008; Mohr et al., 2016). The CRP rise during exercise-induced inflammation has been associated with leukocyte activation and synthesis of adhesion molecules that facilitate leukocyte migration to the injured muscle (Fatouros and Jamurtas, 2016). Soccer match play has been shown to induce leukocytosis and a rise in adhesion molecules in the circulation within the first $24 \mathrm{~h}$ of recovery after a soccer game (Mohr et al., 2016). The rise in CRP is mainly attributed to the action of increased cytokine 6 (IL-6) on liver cells to stimulate the synthesis of acute-phase proteins from amino acids released by muscle due to increased proteolysis (Chang and Bistrian, 1998). CRP seems to increase within the first $24 \mathrm{~h}$ after a soccer game and normalize thereafter (Ispirlidis et al., 2008; Mohr et al., 2016). The CST therefore resulted in a typical acute inflammatory response also seen following soccer games and other intense exercise protocols. The short-lived inflammatory response resulted in deterioration in sprint performance of a magnitude $(4 \%)$ usually seen after simulated $(7 \%)$ and actual (3-5\%) soccer play, indicating a similar development in fatigue (Ispirlidis et al., 2008; Krustrup et al., 2006a, 2011; Mohr et al., 2016).

In conclusion, post-game fatigue development in soccer occurs in multiple muscle groups with knee flexors demonstrating the largest fatigue response. Fatigue and recovery patterns vary between muscle groups and individual players, yet trunk muscles display the overall slowest post-game recovery kinetics. Training status correlates inversely with the degree of post-game muscle damage.

\section{Acknowledgements}

We would like to thank the participants for their commitment and efforts, and the clubs and coaches for their positive support. We would also like to thank Prof. Stefan Grau, Dr. Tobias Hein, Dr. Klavs Madsen, and Jonatan Jungmalm and Andreas Zachrisson for their technical assistance. The study was supported by a grant from the Faroese Research Council (Granskingarráðið).

\section{References}

Andersson H, Raastad T, Nilsson J, Paulsen G, Garthe I, Kadi F. Neuromuscular fatigue and recovery in elite female soccer: effects of active recovery. Med Sci Sports Exerc, 2008; 40: 372-380

Ascensao A, Leite M, Rebelo AN, Magalhäes S, Magalhäes J. Effects of cold water immersion on the recovery of physical performance and muscle damage following a one-off soccer match. J Sports Sci, 2011; 29: 217-225

Ascensão A, Rebelo A, Oliveira E, Marques F, Pereira L, Magalhães J. Biochemical impact of a soccer match - 
analysis of oxidative stress and muscle damage markers throughout recovery. Clin Biochem, 2008; 41: 841-851

Bangsbo J, Marcello laia F, Krustrup P. Metabolic Response and Fatigue in Soccer. International J Sports Physiol Perform, 2007; 2: 111-127

Behm DG, Cappa D, Power GA. Trunk muscle activation during moderate-and high-intensity running. Appl Physiol Nutr Metab, 2009; 34: 1008-1016

Bendiksen M, Bischoff R, Randers MB, Mohr M, Rollo I, Suetta C, Bangsbo J, Krustrup P. The Copenhagen Soccer Test: physiological response and fatigue development. Med Sci Sports Exerc, 2012; 44: 1595-1603

Chatzinikolaou A, Draganidis D, Avloniti A, Karipidis A, Jamurtas AZ, Skevaki CL, Tsoukas D, Sovatzidis A, Theodorou A, Kambas A, Papassotiriou I, Taxildaris K, Fatouros IG. The microcycle of inflammation and performance changes after a basketball match. J Sports Sci, 2014; 32: 870-882

Chatzinikolaou A, Fatouros IG, Gourgoulis V, Avloniti A, Jamurtas AZ, Nikolaidis MG, Douroudos I, Michailidis Y, Beneka A, Malliou P, Tofas T, Georgiadis I, Mandalidis D, Taxildaris K. Time course of changes in performance and inflammatory responses after acute plyometric exercise. J Strength Cond Res, 2010; 24: 1389-1398

Delextrat A, Baker J, Cohen DD, Clarke ND. Effect of a simulated soccer match on the functional hamstringsto-quadriceps ratio in amateur female players. Scand J Med Sci Sports, 2013; 23: 478-486

Draganidis D, Chatzinikolaou A, Avloniti A, Barbero-Alvarez JC, Mohr M, Malliou P, Gourgoulis V, Deli CK, Douroudos, II, Margonis K, Gioftsidou A, Flouris AD, Jamurtas AZ, Koutedakis Y, Fatouros IG. Recovery kinetics of knee flexor and extensor strength after a football match. PLoS One, 2015; 10: e0128072

Fatouros IG, Chatzinikolaou A, Douroudos II, Nikolaidis MG, Kyparos A, Margonis K, Michailidis Y, Vantarakis A, Taxildaris K, Katrabasas I, Mandalidis D, Kouretas D, Jamurtas AZ. Time-course of changes in oxidative stress and antioxidant status responses following a soccer game. J Strength Cond Res, 2010; 24: 3278-3286

Fatouros IG, Jamurtas AZ. Insights into the molecular etiology of exercise-induced inflammation: opportunities for optimizing performance. J Inflamm, 2016; 9: 175

Fransson D, Krustrup P, Mohr M. Running intensity fluctuations cause temporary performance decrements in top-class football Sci Med Footb, 2017; 1: 10-17

Gregson W, Drust B, Atkinson G, Salvo VD. Match-to-match variability of high-speed activities in Premier League soccer. Int J Sports Med, 2010; 31: 237-242

Hawkins RD, Hulse M, Wilkinson C, Hodson A, Gibson M. The association football medical research programme: an audit of injuries in professional football. Brit J Sport Med, 2001; 35: 43-47

Ispirlidis I, Fatouros IG, Jamurtas AZ, Nikolaidis MG, Michailidis I, Douroudos I, Margonis K, Chatzinikolaou A, Kalistratos E, Katrabasas I, Alexiou V, Taxildaris K. Time-course of changes in inflammatory and performance responses following a soccer game. Clin J Sport Med, 2008; 18: 423-431

Krustrup P, Mohr M, Nybo L, Jensen JM, Nielsen JJ, Bangsbo J. The Yo-Yo IR2 test: Physiological response, reliability, and application to elite soccer. Med Sci Sports Exerc, 2006a; 38: 1666-1673

Krustrup P, Mohr M, Steensberg A, Bencke J, Kjaer M, Bangsbo J. Muscle and blood metabolites during a soccer game: Implications for sprint performance. Med Sci Sports Exerc, 2006b; 38: 1165-1174

Krustrup P, Ortenblad N, Nielsen J, Nybo L, Gunnarsson TP, Iaia FM, Madsen K, Stephens F, Greenhaff P, Bangsbo J. Maximal voluntary contraction force, SR function and glycogen resynthesis during the first 72 h after a high-level competitive soccer game. Eur J Appl Physiol, 2011; 111: 2987-2995

Magalhaes J, Rebelo A, Oliveira E, Silva JR, Marques F, Ascensao A. Impact of Loughborough Intermittent Shuttle Test versus soccer match on physiological, biochemical and neuromuscular parameters. Eur J Appl Physiol, 2010; 108: 39-48

Mohr M, Draganidis D, Chatzinikolaou A, Barbero-Alvarez JC, Castagna C, Douroudos I, Avloniti A, 
Margeli A, Papassotiriou I, Flouris AD, Jamurtas AZ, Krustrup P, Fatouros IG. Muscle damage, inflammatory, immune and performance responses to three football games in 1 week in competitive male players. Eur J Appl Physiol, 2016; 116: 179-193

Mohr M, Krustrup P, Bangsbo J. Match performance of high-standard soccer players with special reference to development of fatigue. J Sports Sci, 2003; 21: 519-528

Mohr M, Thomassen M, Girard O, Racinais S, Nybo L. Muscle variables of importance for physiological performance in competitive football. Eur J Appl Physiol, 2016; 116: 251-262

Nedelec M, McCall A, Carling C, Legall F, Berthoin S, Dupont G. The influence of soccer playing actions on the recovery kinetics after a soccer match. J Strength Cond Res, 2014; 28: 1517-1523

Randers MB, Mujika I, Hewitt A, Santisteban J, Bischoff R, Solano R, Zubillaga A, Peltola E, Krustrup P, Mohr M. Application of four different football match analysis systems: A comparative study. J Sports Sci, 2010; 28: 171-182

Shinkle J, Nesser TW, Demchak TJ, McMannus DM. Effect of core strength on the measure of power in the extremities. J Strength Cond Res, 2012; 26: 373-380

Silva JR, Ascensão A, Marques F, Seabra A, Rebelo A, Magalhães J. Neuromuscular function, hormonal and redox status and muscle damage of professional soccer players after a high-level competitive match. Eur J Appl Physiol, 2013; 113: 2193-2201

Varley M, Aughey R. Acceleration profiles in elite Australian soccer. Int J Sports Med, 2012; 34: 282-287

\section{Corresponding author:}

\section{Magni Mohr}

Centre of Health Science

Faculty of Health Sciences

University of the Faroe Islands

Jónas Broncks gøta 25. 3rd floor

Tórshavn, Faroe Islands

Tel.: +298 292270

E-mail: magnim@setur.fo 